\title{
Fatores associados à ideação suicida entre travestis e transexuais assistidas por organizações não governamentais
}

\author{
Factors associated with suicidal ideation among travestis and \\ transsexuals receiving assistance from transgender organizations
}

Glauber Weder dos Santos Silva (http://orcid.org/0000-0002-0570-1944) ${ }^{1}$

Karina Cardoso Meira (http://orcid.org/0000-0002-1722-5703) ${ }^{2}$

Dulcian Medeiros de Azevedo (http://orcid.org/0000-0002-4323-091X) ${ }^{3}$

Romeika Carla Ferreira de Sena (http://orcid.org/0000-0003-1230-3888) ${ }^{1}$

Suerda Lillian da Fonseca Lins (http://orcid.org/0000-0002-2154-968X) ${ }^{1}$

Eder Samuel Oliveira Dantas (http://orcid.org/0000-0002-6595-6105) ${ }^{4}$

Francisco Arnoldo Nunes de Miranda (http://orcid.org/0000-0002-8648-811X) ${ }^{1}$

${ }^{1}$ Direção de Enfermagem, Hospital Giselda Trigueiro. R. Cônego Monte 110 Quintas. 59037-170 Natal RN Brasil.

glauberweder@hotmail.com

${ }^{2}$ Escola de Saúde,

Universidade Federal do Rio Grande do Norte. Natal RN

Brasil.

${ }^{3}$ Departamento de

Enfermagem, Universidade

do Estado do Rio Grande do

Norte. Natal RN Brasil.

${ }^{4}$ Hospital Universitário

Onofre Lopes, Universidade

Federal do Rio Grande do

Norte. Natal RN Brasil

\begin{abstract}
The aim of this study was to determine the prevalence of suicidal ideation and explore associated factors among travestis and transsexuals. A cross-sectional study was conducted between 2015 and 2016 with 22 travestis and 36 transsexuals who were members of the four transgender organizations operating in the state of Rio Grande do Norte, Brazil. Associations between suicidal ideation and the categorical variables were measured using the chi-squared test, Fisher's exact test, and likelihood ratios. Multivariate analysis was performed using robust Poisson regression. The suicidal ideation prevalence rate was $41.4 \%$ (95\%CI 41.3-54.51\%): 13.79\% among travestis (95\%CI 4.91-22.66\%) and $27.61 \%$ in transsexuals (95\%CI 16.08-39.08\%). The prevalence of suicidal ideation was higher among respondents who reported suffering violence at school $(P R=$ 2.05; 95\%CI 1.08-3.87) and those experiencing moderate/severe depression $(P R=3.86 ; 95 \% C I$ 1.51-9.83). The findings suggest that unfavorable school contexts and the presence of depression compromise mental health and contribute to suicidal ideation among travestis and transsexuals. Key words Suicidal ideation, Transgender, Gender-based violence, Self-injurious behavior, Vulnerable populations
\end{abstract}

Resumo Objetivou-se analisar a prevalência e os fatores associados à ideação suicida em travestis e transexuais. Estudo transversal, realizado entre 2015 e 2016 com travestis $(n=22)$ e transexuais $(n=36)$ associadas a quatro organizações civis do Rio Grande do Norte. Avaliou-se a diferença entre as categorias por meio dos testes qui-quadrado, exato de Fisher e de razão de verossimilhança. Realizou-se a análise múltipla por meio da regressão de Poisson com variância robusta. A prevalência de ideação suicida foi de 41,4\% (IC 95\%: 41,3$54,51 \%$ ), sendo de $13,79 \%$ entre travestis (IC 95\%: 4,91-22,66\%) e 27,61\% entre transexuais (IC 95\%: 16,08-39,08\%). Observou-se que apresentaram maior prevalência de ideação suicida os participantes com histórico de violência na escola $(R P=2,05$; IC 95\%: 1,08-3,87) e classificados com niveis depressivos moderado/grave $(R P=3,86$; $I C$ 95\%: 1,51-9,83). Contexto escolar desfavorável e presença de sintomatologia depressiva parecem prejudicar a saúde mental e colaborar com a ideação suicida de pessoas transexuais e travestis.

Palavras-chave Ideação suicida, Pessoas transgêenero, Violência de gênero, Comportamento autodestrutivo, Populações vulneráveis 


\section{Introdução}

O suicídio emerge como prioridade em saúde pública por sua alta transcendência e magnitude. Mundialmente, verifica-se mais de 800 mil suicídios por ano, correspondendo à $1,4 \%$ de todas as causas de mortes (11,4 óbitos por 100 mil habitantes $)^{1}$. Representa a segunda maior causa de morte em indivíduos com 15 a 29 anos $^{2,3}$.

Destaca-se que cerca de $78 \%$ dos suicídios são perpetrados em países de baixa e média rendas. Os maiores coeficientes de mortalidade por suicídio em 2015 foram observados no Sudeste Asiático e na Europa, com taxas variando de 11,9 a 14,0 óbitos por 100 mil habitantes, e as menores no Mediterrâneo Oriental (3,8 a 4,3 óbitos por 100 mil habitantes) $)^{3}$. No entanto, é importante destacar que a magnitude dos coeficientes de mortalidade por essa causa pode estar subestimada devido à subnotificação dos registros de óbitos e à grande proporção de óbitos classificados como evento cuja intenção é indeterminada.

No período de 2011 a 2016, o suicídio no Brasil teve taxa de 5,5 óbitos por 100 mil habitantes, com maior risco no sexo masculino $(8,7 / 100 \mathrm{mil}$ homens), sendo cerca de quatro vezes maior que o coeficiente do sexo feminino (2,4/100 mil mulheres). No mesmo recorte temporal, as notificações dos casos aumentaram 209,5\% em mulheres e 194,7\% entre homens. A ocorrência de lesões autoprovocadas concentrou-se na faixa etária de 10 a 39 anos, tanto em homens $(70,1 \%)$ como em mulheres $(74,4 \%)^{4}$.

Esse problema de saúde pública constitui-se em agravo à saúde multifatorial com a tendência temporal e magnitude dos coeficientes, sendo afetadas pelos contextos político, econômico, social, cultural, religioso e características do sujei$\operatorname{tos}^{1,3,5}$. Nesse contexto, a população de travestis e transexuais encontra-se em maior risco de morte por suicídio do que a população geral, devido à sua exposição a eventos estressores associados ao preconceito que vivenciam diuturnamente ${ }^{6}$.

Relatórios divulgados por organizações não governamentais (ONGs) têm documentado o elevado risco de suicídio e comportamento autoinfligido nesse público. Estudo nos Estados Unidos a partir de dados de uma amostra de indivíduos transgêneros $(\mathrm{n}=6.456)$ evidenciou $41 \%$ de prevalência de tentativas de suicídio nesse grupo, sendo cerca de quatro vezes maior que a observada em lésbicas, gays, bissexuais e população geral ${ }^{7}$.

A ideação suicida (IS) tem sido identificada na literatura como fator de risco para a tentativa de suicídio e o suicídio entre travestis e transexuais $^{8,9}$, caracterizando-se como todo processo desde a idealização até a elaboração da morte autoprovocada. Isto posto, evidenciou-se maiores chances de relato de ideação suicida em adultos transgêneros que vivenciavam integramente a sua identidade de gênero $(\mathrm{OR}=2,68, \mathrm{p}<$ $0,01)$, com histórico de violência física e sexual $(\mathrm{OR}=5,44 ; \mathrm{p}<0,001)$, e experienciaram uma ou duas situações discriminatórias $(\mathrm{OR}=2,09$, $\mathrm{p}<0,05$ e OR $=2,86, \mathrm{p}<0,05$, respectivamente $)^{9}$. Em relação às tentativas de suicídio, foram observadas maiores chances ao longo da vida nos sujeitos com maiores níveis de transfobia internalizada $(\mathrm{OR}=1,18 \text {; IC } 95 \%=1,04-1,33)^{10}$. Tais achados sinalizam a importância e a relevância de se destacar a presença da violência simbólica e estrutural na ocorrência de suicídio e IS nessa população.

No Brasil não foram encontrados artigos que investigassem nenhum aspecto do comportamento suicida entre travestis e transexuais nas bases de dados. Supõe-se que essa realidade esteja relacionada à dificuldade de acesso desses sujeitos ao exercício de cidadania, educação, saúde, mobilidade urbana, renda e emprego, entre outros indicadores econômicos e sociodemográficos em território nacional, uma vez que esse grupo populacional não consta nos dados censitários, $o$ que dificulta a produção de conhecimento sobre esse grupo, bem como sua existência demográfica, bem-estar, qualidade, estilo e condições de vida.

Nesse sentido, investigações científicas e desenvolvimento de técnicas e metodologias para o alcance de indivíduos com identidades travestis e transexuais são necessárias para que essa população se torne visível no contexto nacional. Assim, será possível pensar um modelo de saúde equânime, consonante com a promoção da saúde e a busca da prevenção do suicídio, respaldados pela Política Nacional de Saúde Integral de Lésbicas, Gays, Bissexuais, Travestis e Transexuais (LGBT), pelas Diretrizes Nacionais para Prevenção do Suicídio, pela Agenda de Ações Estratégicas para a Vigilância e Prevenção do Suicídio e Promoção da Saúde no Brasil e a Agenda de Prioridades de Pesquisa do Ministério da Saúde do Brasil, que preveem a análise do impacto de determinantes sociais, como gênero e orientação sexual, na saúde mental e o levantamento qualificado da epidemiologia do suicídio em populações de maior vulnerabilidade.

Frente ao exposto, o presente estudo teve como objetivo estimar a prevalência e os fatores 
associados à ideação suicida em travestis e transexuais assistidos por organizações não governamentais no estado do Rio Grande do Norte.

\section{Método}

Trata-se de um estudo transversal que teve como população travestis e transexuais autodeclaradas, associadas(os) às quatro únicas ONGs de direitos transexuais existentes no estado do Rio Grande do Norte (RN), Brasil. Essas ONGs são sediadas em duas microrregiões do estado e com membros em todo o território estadual. Foram fundadas e organizadas pelo movimento regional de pessoas travestis e transexuais, na luta pelo reconhecimento social e direitos civis. Assim, foram cenários deste estudo: a Associação Potiguar de Travestis e Transexuais na Ação pela Coerência no RN; a Associação das Travestis Encontrando a Atuação e Valorização na Saúde Santa-Cruzense; a Associação de Homens-Trans Potiguares; e a Associação de Travestis Reencontrando a Vida.

Nesta pesquisa, considerou-se transexual ou travesti toda pessoa que assim se autodeterminou por acreditar não existir correspondência - socialmente imposta - entre seu sexo biológico e a identidade de gênero, independentemente de resignação sexual, terapia hormonal ou retificação de prenome no Registro Geral. É importante destacar que participaram do estudo homens-trans (designados mulheres no nascimento mas que se identificam com o gênero masculino), mulheres-trans (designados homens no nascimento mas que se identificam com o gênero feminino) e travestis (categoria político-cultural latina que é designada homem ao nascer, assume papel feminino na vida adulta porém não se reconhece como homem ou mulher, mas um terceiro gênero) ${ }^{11}$; todos genericamente denominados pessoas trans.

No período de realização do estudo, a população assistida por essas ONGs era de 79 pessoas. Os critérios de inclusão foram: (i) ter idade igual ou superior a 18 anos; (ii) auto-identificar-se como pessoa trans. Excluíram-se os candidatos que (i) se associaram a alguma das ONGs durante a coleta de dados; (ii) que não estavam residindo no RN; e (iii) pessoas intersexuais ou auto-idenficadas como não-binárias.

$\mathrm{O}$ arrolamento se deu por reunião formal com as pessoas responsáveis pelas ONGs e disponibilização de lista telefônica dos possíveis participantes, para os quais o pesquisador responsável fez ligações a fim de formalizar o convite. Con- tou-se também com a colaboração e mediação das lideranças na abordagem residencial e telefônica aos sujeitos, e em alguns casos ocorria reunião presencial antes da coleta propriamente dita.

Compuseram este estudo $58(73,42 \%)$ sujeitos, selecionados por conveniência e intencionalmente. Destaca-se que a amostra, apesar de constituída por uma pequena quantidade de participantes, é representativa do universo que se pretende a investigar, ou seja, das pessoas travestis e transexuais assistidas pelas ONGs.

Ressaltamos os seguintes motivos que resultaram em perdas de 21 (26,58\%) participantes, mesmo em condições de elegibilidade, o que aponta para a complexidade da problemática: não atender o telefone $(\mathrm{n}=3 ; 3,80 \%)$; não estar no endereço conhecido e informado $(n=5$; 6,33\%); não comparecer ao local escolhido para a entrevista $(\mathrm{n}=4 ; 5,06 \%)$; recusar-se a participar $(n=6 ; 7,59 \%)$; estar sob efeito de substâncias ilícitas em mais de dois momentos da coleta de dados $(\mathrm{n}=1 ; 1,27 \%)$; além disso, dois $(2,53 \%)$ sujeitos elegíveis cometeram suicídio antes da coleta de dados.

A coleta ocorreu entre novembro de 2015 e junho de 2016, precedida pelos esclarecimentos quanto aos objetivos e procedimentos do estudo, a saber: possibilidade de acompanhamento psicológico pós-coleta no Laboratório de Psicologia da Universidade Federal do Rio Grande do Norte, caso desejasse; encerramento da coleta a qualquer momento; a assinatura do Termo de Consentimento Livre e Esclarecido. Para a coleta de dados sociodemográficos, histórico de saúde, histórico de violência e comportamento suicida, utilizou-se um formulário elaborado pelos pesquisadores, pré-testado com cinco coordenadores locais das ONGs que não integraram a amostra final.

O formulário roteirizado foi respaldado pela literatura sobre a temática e conteve questões com os seguintes grupos de variáveis: (i) dados sociodemográficos - idade, idade que assumiu a identidade de gênero, estado civil, espiritualidade, escolaridade, raça, ocupação, renda, orientação sexual e identidade de gênero; (ii) histórico de saúde - infecções sexualmente transmissíveis, conhecimento da sorologia para o vírus da imunodeficiência humana (HIV), cirurgia de redesignação sexual, uso de silicone industrial líquido, hormonoterapia, acompanhamento profissional na hormonoterapia e uso de substâncias psicoativas lícitas ou ilícitas; (iii) histórico de violência sofrida - vitimização, tipologia, espaciali- 
dade, agressores, expulsão do núcleo familiar em razão da identidade de gênero, respeito integral ao nome social, vergonha de si e da identidade de gênero; (iv) comportamento suicida - histórico de tentativa de suicídio, intensidade da vontade de morrer na última tentativa, tentativa de suicídio ou suicídio na família, tentativa de suicídio ou suicídio entre amigos e amigo travesti ou transexual com comportamento suicida.

Em relação aos instrumentos de avaliação da depressão e de IS optou-se, respectivamente, pelo Inventário de Depressão de Beck (BDI) e pela Escala de Ideação Suicida de Beck (BSI). As escalas Beck são consideradas "padrão ouro" para mensuração das variáveis de interesse em tela, as quais foram validadas para o português em 2001. Na adequabilidade da versão em português, originalmente testou-se a fidedignidade dos instrumentos com coeficiente alfa de Cronbach, alcançando-se excelente consistência interna, variando entre 0,75 e $0,95 \mathrm{em}$ todas as escalas com amostras psiquiátricas e não-psiquiátricas ${ }^{12}$.

As escalas validadas de autorrelato BSI e BDI identificam, respectivamente, presença ou ausência de ideação suicida e a intensidade dos sintomas depressivos em populações clínicas e não-clínicas. A BSI constitui-se por 21 itens, divididos em duas partes, cada item pontuado de $0 \mathrm{a}$ 2 pontos, e os 19 primeiros grupos de afirmativas geram o escore final - os outros dois grupos são meramente informativos. Para fins analíticos, a IS foi considerada presente quando o participante pontuava diferente de 0 os grupos 4 e/ou 5 da primeira parte da escala, caracterizando gravidade da IS, e por conseguinte respondia a toda a escala - primeira e segunda parte, revelando intenção suicida ${ }^{12}$.

O BDI é composto por 21 itens, cada um contém quatro alternativas pontuadas de 0 a 3 pontos, representando graus crescentes de gravidade da depressão. Utilizou-se o seguinte parâmetro para estratificação de sintomas em níveis depressivos: mínimo (0 a 11 pontos), leve (12 a 19 pontos), moderado ( 20 a 35 pontos) e grave $\left(36\right.$ a 63 pontos) ${ }^{12}$.

Os dados originários dos instrumentos foram digitados e mediados no software Microsoft Office Excel, v.2016, com dupla digitação, e posteriormente exportados para testes no pacote estatístico R, v.3.5.1.

Para avaliar a confiabilidade das escalas BSI e BDI na população do estudo, foi medido o coeficiente alfa de Cronbach, obtendo-se valores de 0,85 e 0,93 , respectivamente, o que significa boa consistência interna.
$\mathrm{Na}$ análise bivariada, avaliou-se a existência de diferença estatisticamente significativa entre IS (variável dependente) e as variáveis categóricas por meio do teste qui-quadrado, teste exato de Fisher ou razão de verossimilhança, de acordo com a adequabilidade do teste aos dados. O nível de confiança $\alpha$ empregado foi de $95 \%$.

$\mathrm{Na}$ regressão múltipla pelo modelo de Poisson com variância robusta ${ }^{13}$, a escolha de potenciais fatores associados foi iniciado com análises univariadas. As variáveis significativas ao nível de $20 \%$ foram candidatas iniciais para compor o modelo final ${ }^{13,14}$. Após inclusão e exclusão de variáveis adicionadas na ordem de maior a menor significância com o desfecho, testou-se a significância de interações entre variáveis que se mantiveram ao longo do processo, no qual os modelos foram comparados via critério de informação de Akaike $^{14}$. A escolha do modelo final considerou plausibilidades epidemiológica e biológica, além da significância estatística de 5\%, estimando as associações com base nas razões de prevalência ajustadas e nos respectivos intervalos de confiança de $95 \%$.

O estudo seguiu as normas de pesquisa envolvendo seres humanos em atenção à Resolução 466/2012 do Conselho Nacional de Saúde do Brasil, com submissão à Plataforma Brasil e aprovação pelo Comitê de Ética em Pesquisas com Seres Humanos da Universidade Federal do Rio Grande do Norte.

\section{Resultados}

No presente estudo, a prevalência de IS em travestis e transexuais foi de 41,4\% (IC\% 41,354,51 ), sendo $13,79 \%$ entre as travestis (IC $95 \%$ : 4,91-15,66) e $27,61 \%$ (IC95\%: 16,08-39,08) entre pessoas trans. Destaca-se a maior prevalência entre pessoas trans em comparação às travestis, sendo essa diferença estatisticamente significativa $(p \leq 0,05)$.

A pontuação mínima para o BSI foi 0, e máxima de 21 pontos, com média de 4,71 pontos (desvio padrão $=6,497)$. Em relação ao BDI, observou-se mínimo de 0 e máximo de 39 pontos, com média de 14,6 (desvio padrão = 9,392),

Em relação à caracterização sociodemográfica (Tabela 1), a maioria identificou-se como pessoa transexual $(62,1 \%)$ e orientação sexual heterossexual $(82,8 \%)$; a média de idade foi de 26,78 anos, e mediana de 24,5 anos (desvio padrão $=7,96)$. Majoritariamente, eram pessoas solteiras ou viúvas ( $81 \%)$, que declararam ter es- 
Tabela 1. Variáveis de caracterização sociodemográfica de travestis e transexuais assistidas por organização não governamentais do Rio Grande do Norte - Natal, RN, Brasil, dezembro de 2015 a junho de 2016.

\begin{tabular}{|c|c|c|c|}
\hline \multicolumn{2}{|l|}{ Variáveis } & \multirow{2}{*}{$\frac{\mathbf{n}}{22}$} & \multirow{2}{*}{$\frac{\%}{37,9}$} \\
\hline Identidade de gênero & Travesti & & \\
\hline & Transexual & 36 & 62,1 \\
\hline \multirow[t]{2}{*}{ Orientação sexual } & Hétero & 48 & 82,8 \\
\hline & Não-hétero & 10 & 17,2 \\
\hline \multirow[t]{2}{*}{ Faixa etária* } & $>29$ anos & 18 & 31,0 \\
\hline & $\leq 29$ anos & 40 & 69,0 \\
\hline \multirow[t]{2}{*}{ Faixa etária que assumiu a identidade de gênero $^{\dagger}$} & $\leq 18$ anos & 39 & 67,2 \\
\hline & $\geq 19$ anos & 19 & 32,8 \\
\hline \multirow[t]{2}{*}{ Estado civil } & Casado ou união estável & 11 & 19,0 \\
\hline & Solteiro ou viúvo & 47 & 81,0 \\
\hline \multirow[t]{2}{*}{ Espiritualidade } & Sim & 37 & 63,8 \\
\hline & Não & 21 & 36,2 \\
\hline \multirow[t]{2}{*}{ Escolaridade } & Ensino superior & 13 & 22,4 \\
\hline & Ensino médio ou menos & 45 & 77,6 \\
\hline \multirow[t]{2}{*}{ Cor/raça } & Branco & 18 & 31,0 \\
\hline & Preto/pardo & 40 & 69,0 \\
\hline \multirow[t]{3}{*}{ Ocupação } & Sim & 48 & 82,8 \\
\hline & Aposentado & 01 & 1,7 \\
\hline & Não & 09 & 15,5 \\
\hline \multirow[t]{2}{*}{ Prostituição } & Não & 41 & 70,7 \\
\hline & Sim & 17 & 29,3 \\
\hline \multirow[t]{2}{*}{ Renda $^{\ddagger}$} & > 1 salário mínimo & 23 & 39,7 \\
\hline & < 1 salário mínimo & 35 & 60,3 \\
\hline
\end{tabular}

* De acordo com o recorte etário da Organização Mundial da Saúde para maior risco de suicídio; ${ }^{\dagger}$ de acordo com a classificação do Estatuto da Criança e Adolescente do Brasil para caracterizar crianças/adolescentes e adultos; ${ }^{\ddagger}$ salário mínimo vigente em 2016 no Brasil: R\$ 880,00.

Fonte: Elaborado pelos autores.

piritualidade $(63,8 \%)$ e nível de escolaridade de ensino fundamental ou ensino médio (77,6\%). Entre os que cursaram ou cursavam o ensino superior, apenas três $(5,2 \%)$ declararam formação completa. Em relação à raça/cor, os participantes se autodeclararam em maior proporção pretos e pardos (69\%).

Quanto à ocupação, 29,3\% indicaram a atividade de prostituição; $15,5 \%$ não relataram ocupação; $13,8 \%$ cabelereiras; $13,8 \%$ trabalho doméstico; $3,5 \%$ vendedores; $3,5 \%$ atendentes; $3,5 \%$ professores; $3,5 \%$ empresários; e $13,8 \%$ outros (técnico de enfermagem, cuidador de idosos, assistente de serviços gerais, esteticista, gerente, eletricista, aposentado, autônomo e cozinheira).

A renda média mensal dos participantes correspondeu a $\mathrm{R} \$ 1.522,52$ (desvio padrão = $4.567,276$; mediana $=\mathrm{R} \$ 835,00)$. Um sujeito declarou renda individual de $\mathrm{R} \$ 35.000,00$. Ao se excluir essa renda discrepante em relação aos demais participantes, observou-se renda média de $\mathrm{R} \$ 935,19$ (mediana $=\mathrm{R} \$ 800,00$ ).
$\mathrm{Na}$ população do estudo não se evidenciou diferença significativa entre o desfecho IS (sim/ não) e os dados sociodemográficos e de histórico de saúde (Tabela 2). No que se refere à avaliação dos níveis e sintomas de gravidade depressiva, houve diferença estatisticamente significativa, com IS $(p=0,002)$ - os participantes que não apresentaram IS tinham maior proporção de níveis depressivos mínimo/leve quando comparados aos que apresentavam ideação suicida.

Entre as características do histórico de violência (Tabela 3), observou-se diferença estatisticamente significativa entre IS e espacialidade escolar da violência perpetrada $(\mathrm{p}=0,012)$. Constatou-se que os indivíduos com ideação suicida relataram maior frequência de violência no ambiente escolar em comparação aos sem IS $(25,9 \%$ versus $17,2 \%)$. Também se verificou maior proporção de ideação suicida entre os sujeitos que não relataram expulsão do núcleo familiar em razão da identidade de gênero $(36,2 \%$ versus $5,2 \% ; \mathrm{p}=0,020)$. 
Tabela 2. Distribuição dos dados sociodemográficos, histórico de saúde e níveis de depressão de travestis e transexuais segundo a presença ou ausência de ideação suicida - Natal, RN, Brasil, dezembro de 2015 a junho de 2016.

\begin{tabular}{|c|c|c|c|c|c|c|}
\hline \multirow{3}{*}{ Variáveis } & & \multicolumn{4}{|c|}{ Ideação suicida } & \multirow{3}{*}{ p-valor ${ }^{\star}$} \\
\hline & \multirow{2}{*}{$\begin{array}{l}\text { Presente } \\
\text { n }\end{array}$} & \multicolumn{3}{|c|}{ Ausente } & & \\
\hline & & $\%$ & $\mathbf{n}$ & $\%$ & & \\
\hline \multirow[t]{2}{*}{ Identidade de gênero } & Travesti & 14 & 24,1 & 8 & 13,8 & 0,544 \\
\hline & Transexual & 20 & 34,5 & 16 & 27,6 & \\
\hline \multirow[t]{2}{*}{ Orientação sexual } & Hétero & 26 & 44,8 & 22 & 37,9 & $0,171^{\dagger}$ \\
\hline & Não-hétero & 8 & 13,8 & 2 & 3,5 & \\
\hline \multirow[t]{2}{*}{ Faixa etária } & $>29$ anos & 22 & 37,9 & 18 & 31,1 & 0,403 \\
\hline & $\leq 29$ anos & 12 & 20,7 & 6 & 10,3 & \\
\hline \multirow{2}{*}{$\begin{array}{l}\text { Faixa etária que assumiu a } \\
\text { identidade de gênero }\end{array}$} & $\leq 18$ anos & 21 & 36,2 & 18 & 31,1 & 0,29 \\
\hline & $\geq 19$ anos & 13 & 22,4 & 6 & 10,3 & \\
\hline \multirow[t]{2}{*}{ Estado civil } & Casado ou união estável & 26 & 44,8 & 21 & 36,2 & $0,333^{\dagger}$ \\
\hline & Solteiro ou viúvo & 8 & 13,8 & 3 & 5,2 & \\
\hline \multirow[t]{2}{*}{ Espiritualidade } & Sim & 25 & 43,1 & 12 & 20,7 & 0,066 \\
\hline & Não & 9 & 15,5 & 12 & 20,7 & \\
\hline \multirow[t]{2}{*}{ Escolaridade } & Ensino superior & 26 & 44,8 & 19 & 32,8 & 0,808 \\
\hline & Ensino médio ou menos & 8 & 13,8 & 5 & 8,6 & \\
\hline \multirow[t]{2}{*}{ Cor/raça } & Branco & 8 & 13,8 & 10 & 17,2 & 0,141 \\
\hline & Preto/pardo & 26 & 44,8 & 14 & 24,2 & \\
\hline \multirow[t]{2}{*}{ Empregabilidade } & Sim ou aposentado & 28 & 48,3 & 20 & 34,5 & $0,999^{\dagger}$ \\
\hline & Não & 6 & 10,3 & 4 & 6,9 & \\
\hline \multirow[t]{2}{*}{ Prostituição } & Não & 24 & 41,4 & 17 & 29,3 & 0,983 \\
\hline & Sim & 10 & 17,2 & 7 & 12,1 & \\
\hline \multirow{2}{*}{ Renda } & > 1 salário mínimo & 13 & 22,4 & 10 & 17,2 & 0,792 \\
\hline & $<1$ salário mínimo & 21 & 36,2 & 14 & 24,1 & \\
\hline \multirow{2}{*}{$\begin{array}{l}\text { Infecções sexualmente } \\
\text { transmissíveis na vida }\end{array}$} & Não & 25 & 43,1 & 19 & 32,8 & 0,621 \\
\hline & Sim & 9 & 15,5 & 5 & 8,6 & \\
\hline \multirow{2}{*}{$\begin{array}{l}\text { Conhecimento da sorologia } \\
\text { positiva para } \mathrm{HIV}^{\ddagger}\end{array}$} & Não & 29 & 50 & 23 & 39,7 & $0,384^{\dagger}$ \\
\hline & Sim & 5 & 8,6 & 1 & 1,7 & \\
\hline \multirow[t]{2}{*}{ Cirurgia de residignação sexual ${ }^{\S}$} & Não & 34 & 58,6 & 24 & 41,4 & - \\
\hline & Sim & 0 & 0 & 0 & 0 & \\
\hline \multirow{2}{*}{$\begin{array}{l}\text { Presença corporal de silicone } \\
\text { industrial líquido }\end{array}$} & Não & 19 & 32,8 & 13 & 22,4 & 0,897 \\
\hline & Sim & 15 & 25,9 & 11 & 19 & \\
\hline \multirow[t]{2}{*}{ Hormonoterapia } & Não & 6 & 10,3 & 7 & 12,1 & 0,3 \\
\hline & Sim & 28 & 48,3 & 17 & 29,3 & \\
\hline \multirow{2}{*}{$\begin{array}{l}\text { Acompanhamento profissional } \\
\text { na hormonoterapia }\end{array}$} & Não & 8 & 13,8 & 11 & 19 & 0,074 \\
\hline & Sim & 26 & 44,8 & 13 & 22,4 & \\
\hline \multirow{2}{*}{$\begin{array}{l}\text { Uso de substâncias psicoativas } \\
\text { lícitas ou ilícitas }\end{array}$} & Não & 6 & 10,3 & 1 & 1,7 & $0,220^{\dagger}$ \\
\hline & Sim & 28 & 48,3 & 23 & 39,7 & \\
\hline \multirow[t]{2}{*}{ Níveis depressivos } & Mínimo/leve & 30 & 51,7 & 12 & 20,7 & $0,002^{\dagger}$ \\
\hline & Moderado/grave & 4 & 6,9 & 12 & 20,7 & \\
\hline
\end{tabular}

${ }^{\star}$ p-valor: teste qui-quadrado; ${ }^{\dagger}$ teste exato de Fisher; ${ }^{\ddagger}$ vírus da imunodeficiência humana; ${ }^{\S}$ o teste de associação não foi realizado porque a variável “cirurgia de redesignação sexual” foi uma constante.

Fonte: Elaborado pelos autores.

Em relação ao comportamento suicida (Tabela 4), evidenciou-se que os indivíduos com histórico pregresso de tentativa de suicídio apresentaram maior proporção de IS (22,4\% versus 19,0 , $\mathrm{p}=0,008)$. Além disso, os indivíduos que nunca tentaram suicídio ou que apresentaram intensidade fraca da vontade de morrer na última tentativa de suicídio apresentaram maior prevalência de IS quando comparados aos de intensidade moderada ou forte $(15,5 \%$ versus $6,9 \%, \mathrm{p}=0,028)$. 
Tabela 3. Distribuição do histórico de violência entre travestis e transexuais segundo a presença ou ausência de ideação suicida - Natal, RN, Brasil, dezembro de 2015 a junho de 2016.

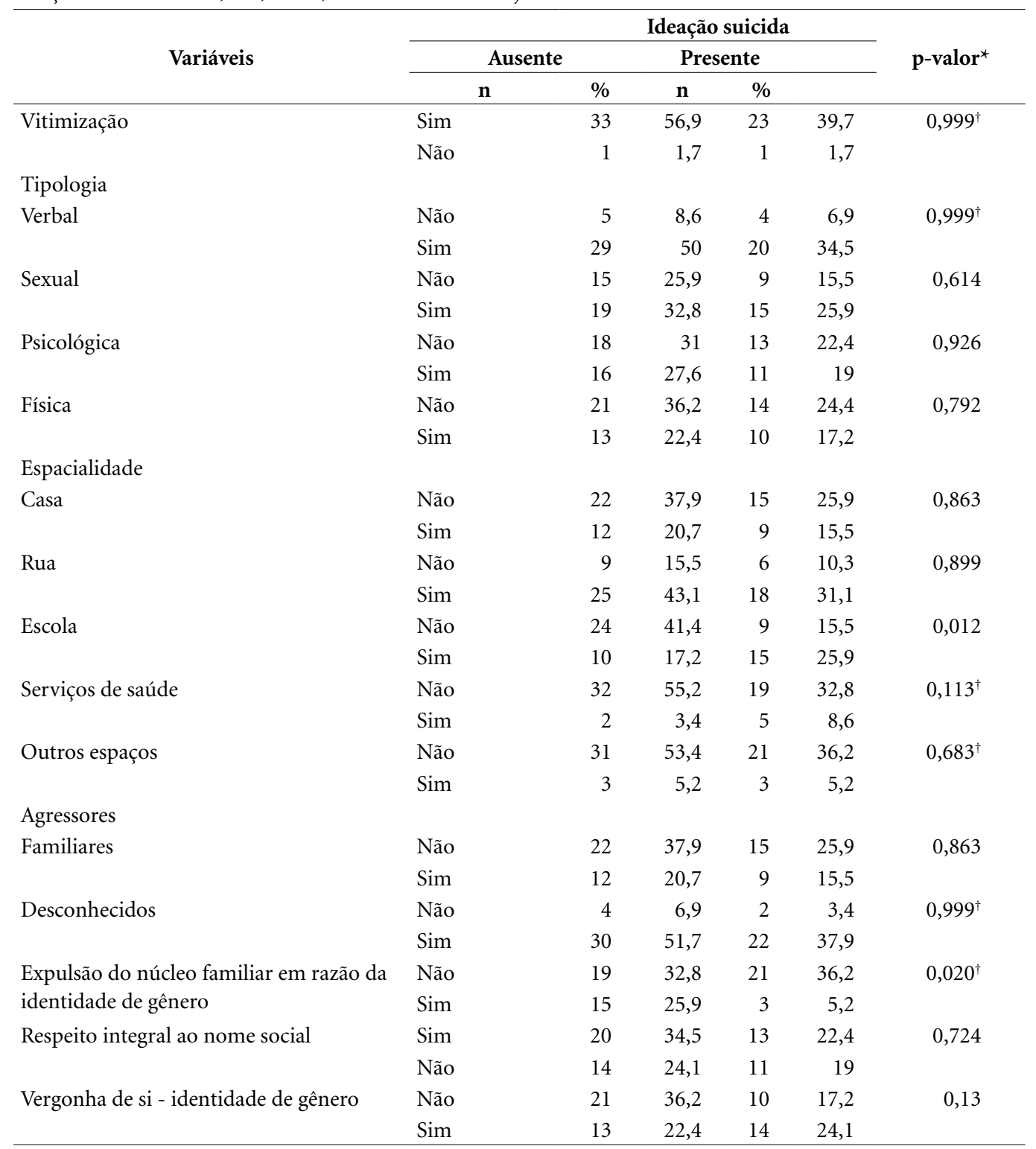

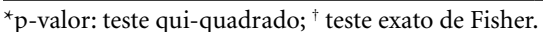

Fonte: Elaborado pelos autores.

$\mathrm{Na}$ análise múltipla (Tabela 5), observou-se que os participantes com histórico de violência na escola $(\mathrm{RP}=2,05$; IC 95\%: $1,08-3,87)$ e classificados com níveis depressivos moderado/grave $(\mathrm{RP}=3,86$; IC 95\%: 1,51-9,83) apresentaram maior prevalência de ideação suicida.

\section{Discussão}

Esta pesquisa evidenciou alta prevalência de IS entre travestis e transexuais assistidas por orga- nizações não governamentais no estado do Rio Grande do Norte, associando-se a espiritualidade, níveis depressivos, violência no ambiente escolar, histórico de tentativa pregressa de suicídio e expulsão do núcleo familiar em razão da identidade de gênero.

Identificou-se prevalência de $41,4 \%$ de IS nos últimos 15 dias que antecederam a coleta de dados, sendo maior em pessoas trans do que em travestis. Essa prevalência foi inferior à encontrada em estudo realizado nos EUA, no qual a proporção de ideação suicida identifica- 
Tabela 4. Distribuição do comportamento suicida entre travestis e transexuais segundo a presença ou ausência de ideação suicida - Natal, RN, Brasil, dezembro de 2015 a junho de 2016.

\begin{tabular}{|c|c|c|c|c|c|c|}
\hline \multirow{3}{*}{ Variáveis } & & \multicolumn{4}{|c|}{ Ideação suicida } & \multirow{3}{*}{ p-valor ${ }^{*}$} \\
\hline & \multirow[t]{2}{*}{ Ausente } & \multicolumn{4}{|c|}{ Presente } & \\
\hline & & $\mathbf{n}$ & $\%$ & $\mathbf{n}$ & $\%$ & \\
\hline \multirow{2}{*}{$\begin{array}{l}\text { Histórico de tentativa pregressa de } \\
\text { suicídio }\end{array}$} & Não & 27 & 46,6 & 11 & 19,0 & \multirow[t]{2}{*}{0,008} \\
\hline & Sim & 7 & 12,1 & 13 & 22,4 & \\
\hline \multirow[t]{2}{*}{$\begin{array}{l}\text { Intensidade da vontade de morrer na } \\
\text { última tentativa }\end{array}$} & $\begin{array}{l}\text { Nunca tentou ou intensidade } \\
\text { fraca }\end{array}$ & 30 & 51,7 & 15 & 25,9 & \multirow[t]{2}{*}{$0,028^{\dagger}$} \\
\hline & Intensidade moderada ou forte & 4 & 6,9 & 9 & 15,5 & \\
\hline \multirow[t]{2}{*}{ Comportamento suicida na família } & Não & 27 & 46,6 & 20 & 34,5 & \multirow[t]{2}{*}{$0,999^{\dagger}$} \\
\hline & Sim & 7 & 12,1 & 4 & 6,9 & \\
\hline \multirow[t]{2}{*}{ Comportamento suicida entre amigos } & Não & 5 & 8,6 & 5 & 8,6 & \multirow[t]{2}{*}{0,543} \\
\hline & Sim & 29 & 50 & 19 & 32,8 & \\
\hline \multirow{2}{*}{$\begin{array}{l}\text { Amigo com comportamento suicida } \\
\text { de identidade travesti ou transexual }\end{array}$} & Não & 13 & 22,4 & 11 & 19,0 & \multirow[t]{2}{*}{0,562} \\
\hline & Sim & 21 & 36,2 & 13 & 22,4 & \\
\hline
\end{tabular}

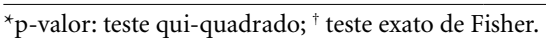

Fonte: Elaborado pelos autores.

Tabela 5. Associação entre as variáveis níveis depressivos, histórico de violência e ideação suicida entre travestis e transexuais assistidas por organizações não governamentais no estado do Rio Grande do Norte, em um modelo de regressão lógica múltipla - Natal, RN, Brasil, dezembro de 2015 a junho de 2016.

\begin{tabular}{|c|c|c|}
\hline Variáveis & $\mathrm{RP}^{\star}$ não ajustada $\left(\mathrm{IC}^{\dagger}\right.$ 95\%) & $\mathrm{RP}^{\star}$ ajustada $\left(\mathrm{IC}^{\dagger}\right.$ 95\%) \\
\hline \multicolumn{3}{|l|}{ Níveis depressivos } \\
\hline Mínima/leve & \multicolumn{2}{|c|}{$1^{\ddagger}$} \\
\hline Moderada/grave & $1,36(1,16-1,60)$ & $3,86(1,51-9,83)$ \\
\hline \multicolumn{3}{|c|}{ Espacialidade da violência - escola } \\
\hline Não & \multicolumn{2}{|c|}{$1^{\ddagger}$} \\
\hline Sim & $1,28(1,06-1,49)$ & $2,05(1,08-3,87)$ \\
\hline
\end{tabular}

* Razão de prevalência; ${ }^{\dagger}$ intervalo de confiança; ${ }^{\ddagger}$ categoria de referência.

Fonte: Elaborado pelos autores.

da foi de $64,9 \%{ }^{9}$. As disparidades apresentadas na prevalência de IS podem estar relacionadas a diferenças metodológicas e a distintos tipos de instrumentos utilizados. Porém, reforça-se a exploração do tema e o uso de instrumental para maior aprofundamento da problemática, tal qual a contribuição prevista nas diretrizes nacionais para prevenção do suicídio no que diz respeito a contribuir para o desenvolvimento de métodos, a coleta de dados e a disseminação de informações e conhecimentos ${ }^{15}$.

Em relação à população geral, observa-se maior risco de ideação, tentativas e suicídio na população de travestis e pessoas transgênero. Essa conclusão resulta de uma revisão sistemáti$\mathrm{Ca}^{16}$ que averiguou as estruturas de ideação suicida entre esses grupos. Cenário corroborado por um estudo de revisão de literatura ${ }^{17}$ que apontou
$77 \%$ de ideação suicida e $43 \%$ de tentativa de suicídio em pessoas trans, sendo que na população geral, no mesmo período, essas taxas foram de $0,5 \%$ e $3,7 \%$, respectivamente.

A associação entre IS e nível depressivo evidenciada no presente estudo está em consonância com estudo realizado em Ontário, Canadá, que aponta que pessoas vivendo na fronteira de gênero $(\mathrm{n}=704)$ apresentavam elevadas pontuações para depressão e maior possibilidade de desenvolver depressão nos 12 meses anteriores à coleta de dados, quando comparadas a mulheres em concordância com a identidade de gênero e o sexo biológico (cisgênero) $(\mathrm{p}=0,009)^{18}$.

As diferenças de gênero permanecem em todo o ciclo de vida. Um estudo, por exemplo, observou que $48 \%$ das pessoas trans idosas apresentavam sintomatologia depressiva ${ }^{19}$, enquanto 
$15 \%$ da população geral de idosos mostrou algum sintoma depressivo ${ }^{20}$. Acredita-se que esta realidade se relaciona ao estigma e à discriminação sofridos pela população travesti e transexual, sendo o transtorno depressivo desencadeado por fatores como idade avançada, baixa autoestima, pior função interpessoal, menor apoio social ${ }^{21} \mathrm{e}$ violência causada por parceiro íntimo ${ }^{22}$.

Nesse sentido, a depressão estaria relacionada ao ciclo de violências que perpetua a exclusão e a disfunção social, a marginalização do segmento, o tratamento injusto, assédios e rejeições. Esse contexto é bem demonstrado no Modelo de Estresse de Minoria proposto por $\mathrm{Meyer}^{23}$, que demonstra que populações minoritárias com indivíduos socialmente desvalorizados são mais expostas a maus-tratos e discriminações crônicas, o que pode gerar baixa autoavaliação, baixo autoconceito, baixa autoestima, status de estigmatização ocultado e expectativa de rejeição. Esses estressores podem levar à má adaptação em processos de adoecimento e a maus resultados de saúde, inclusive mental.

Tratando-se da violência, a espacialidade escolar e a expulsão do núcleo familiar constituem importantes achados desta pesquisa, especificamente como aspectos associados à IS. Caracterizada como preditor de comportamento suicida entre vários grupos, a violência circunscreve-se como elemento marcante na vida de travestis e transexuais no Brasil ${ }^{24}$, desempenhando papel fundamental na subjetividade desses indivíduos. Tal percepção da violência como risco para suicídio é endossada pela agenda nacional de prioridades para prevenção ao suicídio ${ }^{25}$, no tópico “educação permanente", inserindo o tema na qualificação dos profissionais de saúde de forma a evidenciar a interface da discriminação e o fenômeno do suicídio.

No presente estudo, travestis e transexuais que relataram violência no âmbito escolar tiveram maior prevalência de ideação suicida. Essa realidade alcança outros contextos espaciais. Por exemplo, na pesquisa bienal de crianças saudáveis e de estudantes da Califórnia ${ }^{26}$, a prevalência de ideação suicida entre jovens trans nos últimos 12 meses anteriores à investigação foi duas vezes maior do que nos demais estudantes $(33,73 \%$, p $<0,001$ ), e tiveram 2,99 vezes mais chances de relatar ideação suicida no último ano escolar (IC $95 \%=2,25-3,98)$. Em estudo ${ }^{27}$ realizado na Nova Zelândia, estudantes transexuais tinham sua saúde e seu bem-estar comprometidos em razão do bullying escolar ( $\mathrm{OR}=4,5$; IC 95\% = 2,4-8,2). No Brasil, o "Estudo Muriel”28, realizado em São
Paulo, observou que ter menor escolaridade (coeficiente de Pearson $(\mathrm{CP})=-4,1, \mathrm{p}<0,05)$ e ter sofrido violência verbal $(\mathrm{CP}=-8,5 ; \mathrm{p}<0,05)$ ou sexual $(C P=-7,8 ; p<0,05)$ estavam associados ao menor bem-estar psicológico dessa população.

Contextualmente, faz-se necessário e relevante destacar que a pessoas travestis e transexuais estão mais expostas à violência por sua assimetria/ distanciamento do ideal patriarcal de heteronormatividade para masculinidade e feminilidade, abandonando, muitas vezes, ou sendo expulsos da escola, fatores que contribuem para a marginalização do segmento e a baixa profissionalização ${ }^{29}$. Corroborando essa hipótese, 20\% dos participantes do presente estudo desempenhavam atividades relacionadas à prostituição, realidade que pode ser consequência da violência escolar, assim como aumentar a exposição à violência.

Nesse sentido, é importante destacar que a superação da violência escolar e o acesso ao ensino de nível superior nessa população constitui-se como fator de proteção para as tentativas de suicídio ao longo da vida, como se nota ao comparar os índices destes com os dos travestis e pessoas trans com escolaridade até ensino médio (OR $=0,49 ;$ IC 95\%: 0,31-0,75) ${ }^{10}$.

As travestis e, consequentemente, as pessoas transexuais também começam a vivenciar experiências de agressão e discriminação desde o momento que se permitem exteriorizar sua identidade de gênero. Assim, no âmbito das espacialidades, análogo ao ambiente escolar, a descoberta do gênero é o momento no qual são vítimas de violência física e verbal no contexto familiar. Não estar nos padrões de gênero esperados pela família é um dos primeiros obstáculos, sórdidos e desumanos, vivenciados por essas pessoas ${ }^{29}$.

Nesta investigação, as travestis e transexuais que estavam no ambiente doméstico demonstraram maior proporção de ideação suicida quando comparadas com aquelas que tinham sido expulsas do núcleo familiar. Tal realidade pode estar relacionada aos desajustes e às instabilidades familiares que têm sido relatados na literatura científica como fatores de risco para a tentativa ou a consumação de suicídio ${ }^{30-32}$. Em contrapartida, o suporte familiar foi identificado como fator de proteção para IS (risco relativo $=0,43$; IC 95\%: $0,26,-0,73)^{33}$

É importante destacar que a variável tentativa pregressa de suicídio esteve associada à ideação suicida, com a maior prevalência entre os que foram classificados com ideação suicida. No presente estudo, a prevalência de tentativa de suicídio foi de 34,5\%, assemelhando-se à observada em uma 
pesquisa com pessoas transgênero vivendo nos Estados Unidos $(32,36 \%)^{10} \mathrm{e}$ em estudo realizado na Argentina $(33 \%)^{34}$, porém diferindo do evidenciado em outra investigação nos EUA sobre comportamento suicida entre transgêneros $(41 \%)^{7}$.

Nessa direção, estudo sobre a saúde e o bem -estar de estudantes neozelandeses subdivididos em grupos de gênero evidenciou que 19,8\% dos transgêneros (OD = 5,0; IC 95\% = 2,9-8,8) haviam tentado suicídio nos últimos 12 meses antes da pesquisa, contra $4,1 \%$ entre os estudantes não transgêneros ${ }^{27}$, prevalência inferior à encontrada neste estudo. Em outra pesquisa nos Estados Unidos, $32,4 \%$ já haviam tentado pelo menos uma vez, e em $6,41 \%$ a tentativa tinha ocorrido nos últimos 12 meses $^{10}$.

Esses achados reafirmam a tentativa de suicídio como um ato comum no cotidiano das minorias de gênero, associada ao conjunto de situações cotidianas vexatórias, preconceituosas, discriminatórias e violentas às quais estão expostas, promovendo sentimentos e sintomas de tristeza profunda, melancolia e egodistonia. Como previsto nas estratégias e políticas para a prevenção do suicídio no Brasil, torna-se necessário a sensibilização e mobilização do campo da saúde e demais setores estatais, bem como da sociedade civil, para atuar nas determinantes sociais relacionadas ao suicídio e sua interseção com a identidade de gênero, compreendendo-se que toda a sociedade tem responsabilidade sobre as mortes voluntárias que produz ${ }^{15,25}$.

Por fim, há de se considerar como limitação do estudo o fato de não se ter investigado uma amostra populacional de pessoas travestis e transexuais no estado do RN, não por uma falha no processo e planejamento do estudo, mas pela inexistência de parâmetros identificadores dessa população, dado não ser contabilizada nos inquéritos censitários, além do próprio acesso dela no contexto social e suas dimensões de vida. Entretanto, considera-se viável, em estudos posteriores, a utilização da técnica de amostragem por bola de neve, considerada importante para o arrolamento de populações escondidas ou de difícil acesso, facilitando o contato e sua identificação ${ }^{28}$.

Também é importante destacar as limitações dos estudos transversais, em que o desfecho e as variáveis independentes são coletados ao mesmo tempo, sendo sujeitos aos vieses de causalidade reversa e de sobrevivência. Assim, para a produção de outras evidências com diferentes abordagens, o desenvolvimento de pesquisas qualitativas pode desvendar relações simbólicas das pessoas trans com o objeto suicídio (repre- sentações sociais) ou identificar processos sociais básicos que perpetuam a violência social contra travestis e transexuais (teoria fundamentada nos dados), explicitando como esse comportamento está relacionado a escolhas individuais e coletivas, assim como a sujeição dessas pessoas à sociedade patriarcal e heteronormativa no controle de seus corpos.

Eleva-se a importância dos dados apresentados por representarem a totalidade de travestis e transexuais associadas civilmente no $\mathrm{RN}$, estudo pioneiro que dimensiona uma identidade organizativa/classe, sobretudo de pertencimento a um grupo considerado vulnerável, além de provocar a discussão acerca da prevenção de suicídio e da promoção da saúde mental em populações vulneráveis, ainda invisíveis ou prejudicadas na oferta e/ou acesso a serviços públicos (saúde, assistência social, lazer etc.).

Frente aos resultados ora apresentados, destacam-se a responsabilidade e as ferramentas que a saúde coletiva possui para liderar o caminho na criação de espaços seguros e inclusivos para essa população, objetivando a prevenção e o manejo da ideação suicida. Recomenda-se aos profissionais de saúde propor intervenções voltadas para a saúde na transgeneridade, principalmente no contexto escolar enquanto espaço de sociabilidade, podendo assim dirimir a magnitude de sintomas depressivos por mal ajustamento social.

Em nível estrutural e político, nossos resultados podem orientar e fomentar, progressivamente, o reconhecimento do conceito de gênero e educação sexual no planejamento do Plano Nacional de Educação, ao evidenciar o espaço escolar como lócus de violência contra pessoas trans, contrapondo-se ao discurso da "ideologia de gênero" que interdita e silencia vozes transexuais nas escolas. Além disso, são recomendadas estratégias de promoção da saúde mental e de prevenção do suicídio, consonantes aos apontamentos dos manuais da Organização Mundial da Saúde ("Prevenindo a violência juvenil: um panorama das evidências") ${ }^{35} \mathrm{e}$ do Ministério da Saúde do Brasil ("Agenda de ações estratégicas para vigilância e prevenção do suicídio e promoção da saúde no Brasil: 2017 a 2020") $)^{25}$, além de redefinir papéis e funções nas políticas públicas de educação e saúde para a diversidade.

\section{Conclusão}

Evidenciou-se que pessoas travestis e transexuais assistidas pelas quatro ONGs no Rio Grande do 


\section{Colaboradores}

GWS Silva trabalhou na concepção, delineamento, análise e interpretação dos dados, na redação do artigo, sua revisão crítica e aprovação da versão a ser publicada; KM Cardoso contribuiu na análise e interpretação dos dados, na redação do artigo, sua revisão crítica e aprovação da versão final; DM Azevedo trabalhou na análise e interpretação dos dados, na redação do artigo, sua revisão crítica e na aprovação da versão a ser publicada; RCF Sena contribuiu na análise e interpretação dos dados, na redação do artigo, sua revisão crítica e aprovação da versão final; SLF Lins participou da análise e interpretação dos dados, da redação do artigo, sua revisão crítica e aprovação da versão a ser publicada; ESO Dantas trabalhou na análise e interpretação dos dados, na redação do artigo, sua revisão crítica e aprovação da versão final; FAN Miranda contribuiu na concepção, delineamento, análise e interpretação dos dados, na redação do artigo, sua revisão crítica e aprovação da versão a ser publicada.

\section{Agradecimentos}

A Rebecka de França, Jacqueline Brasil, Lara Biank e Nathan Philipe, pelo apoio no arrolamento dos participantes da pesquisa.

\section{Financiamento}

Este artigo é fruto da dissertação de Mestrado em Enfermagem do autor principal, que teve financiamento da Coordenação de Aperfeiçoamento de Pessoal de Nível Superior (Capes) por meio de bolsa de mestrado. tação de políticas que objetivem o controle da IS e seus agravantes. $O$ caráter associativo e coletivo dos participantes da pesquisa em ONGs pode ter conferido um fator protetivo para o desenvolvimento e rastreamento da ideação suicida, sendo possivel que entre indivíduos não associados os resultados sejam exponencialmente alarmantes. Recomenda-se a realização de outras investigações que alcancem recortes populacionais e delineamentos metodológicos na transgeneridade.

\section{Referências}

1. Júnior AF. The suicidal behavior in Brazil and in the world. Revista Brasileira de Psicologia 2015; 2:15-28.

2. World Health Organization (WHO). Preventing suicide: a global imperative. Geneva: WHO; 2014.

3. Bachmann S. Epidemiology of suicide and the psychiatric perspective. Int J Environ Res Public Health 2018; 15(7):e1425.

4. Secretaria de Vigilância Epidemiológica. Suicídio: saber, agir e prevenir. Brasília: Ministério da Saúde; 2017.

5. Fountoulakis KN, Kawohl W, Theodorakis PN, Kerkhof AJ, Navickas A, Hoschl C, Lecic-Tosevski D, Sorel E, Rancans E, Palova E, Juckel G, Isacsson G, Jagodic HK, Botezat-Antonescu I, Warnke I, Rybakowski J, Azorin JM, Cookson J, Waddington J, Pregelj P, Demyttenaere K, Hranov LG, Stevovic LI, Pezawas L, Adida M, Figuera ML, Pompili M, Jakovljevi M, Vichi M, Perugi G, Andreassen O, Vukovic O, Mavrogiorgou P, Varnik P, Bech P, Dome P, Winkler P, Salokangas RK, From T, Danileviciute V, Gonda X, Rihmer Z, Benhalima JF, Grady A, Leadholm AK, Soendergaard S, Nordt C, Lopez-Ibor J. Relationship of suicide rates to economic variables in Europe: 2000-2011. Br J Psychiatric 2014; 205(6):486-496.

6. Caravaca-Morera JA, Padilha MI. Bodies in motion: spaces, emotions and representations that (de)construct realities. Rev Esc Enferm USP 2017; 51(1):e03203.

7. Haas AP, Rodgers PL, Herman JL. Suicide attempts among transgender and gender non-conforming adults: findings of the national transgender discrimination survey. Los Angeles: The Williams Institute/ American Foundation for Suicide Prevention; 2014.

8. Mereish EH, O'Cleirigh C, Bradford JB. Interrelationships between LGBT-based victimization, suicide, and substance use problems in a diverse sample of sexual and gender minority men and women. Psychol Health Med 2014; 19(1):1-13.

9. Rood BA, Puckett JA, Pantalone DW, Bradford JB. Predictors of suicidal ideation in a Statewide sample of transgender individuals. LGBT Health 2015; 2(3):270-275. 
10. Perez-Brumer A, Haltzenbuehler ML, Oldenburg CE, Bockting W. Individual-and structural-level risk factors for suicide attempts among transgender adults. Behav Med 2015; 41(3):164-171.

11. Kulick D. Travesti: prostituição, sexo, gênero e cultura no Brasil. Rio de Janeiro: Editora Fiocruz; 2008.

12. Cunha JA. Manual da versão em português das Escalas Beck. São Paulo: Casa do Psicólogo; 2016.

13. Coutinho LMS, Scazufca M, Menezes PR. Methods for estimating prevalence ratios in cross-sectional studies. Rev Saude Publica 2008; 42(6):992-998.

14. Hosmer DW, Lemeshow S. Applied logistic regression. New York: John Wiley and Sons; 2000.

15. Brasil. Ministério da Saúde. Gabinete do Ministro. Portaria no 1876 , de 14 de agosto de 2006. Institui Diretrizes Nacionais para Prevenção do Suicídio. Brasília (DF): Ministério da Saúde; 2006.

16. Wolford-Clevenger C, Frantell K, Smith PN, Flores LY, Stuart GL. Correlates of suicide ideation and behaviors among transgender people: a systemic review guided by ideation-to-action theory. Clin Psych Review 2018; 63:93-105.

17. Adams N, Hitomi M, Moody C. Varied reports of adult Transgender Suicidality: Synthesizing and Describing the Peer-Reviewed and Gray Literature. Transgender Health 2017; 2(1):60-75.

18. Williams CC, Curling D, Steele LS, Gibson MF, Daley A, Green DC, Ross LE. Depression and discrimination in the lives of women, transgender and gender liminal people in Ontario, Canada. Health Soc Care Community 2017; 25(3):1139-1150.

19. Hoy-Ellis CP, Fredriksen-Goldsen KI. Depression among transgender older adults: general and minority stress. Am J Community Psychol 2017; 59(3-4):0-11.

20. Lima AMP, Ramos JLS, Bezerra IMP, Rocha RPB, Batista HMT, Pinheiro WR. Depression in the elderly: a systematic review of the literature. Revista de Epidemiologia e Controle de Infecção 2016; 6:97-103.

21. Witcomb GL, Bouman WP, Claes L, Brewin N, Crawford JR, Arcelus J. Levels of depression in transgender people and its predictors: results of a large matched control study with transgender people accessing clinical services. J. Affect Disord 2018; 235:308-315.

22. Bukowski LA, Hampton MC, Escobar-Vieira CG, Sang JM, Chandler CJ, Henderson E, Creasy SL, Stall RD. Intimate partner violence and depression among black transgender women in the USA: the potential suppressive effect of perceived social support. J Urban Health 2019; 22:1-12.

23. Gamarel KE, Reisner SL, Laurenceau JP, Nemoto T, Operario D. Gener minority stress, mental health and relationship quality: a dyadic investigation of transgender women and their cisgender partners. J Fam Psychol 2014; 28(4):437-447.

24. Silva GWS, Souza EFL, Sena RCF, Moura IBL, Sobreira MVS, Miranda FAN. Cases of violence involving transvestites and transsexuals in a northeastern Brazilian city. Rev Gauch Enferm 2016; 37(2):e56407.

25. Ministério da Saúde (MS). Agenda de ações estratégicas para a vigilância e prevenção do suicídio e promoção da saúde no Brasil. Brasília: MS; 2017.
26. Perez-Brumer A, Day JK, Russell ST, Haltzenbuehler ML. Prevalence and correlates of suicidal ideation among transgender youth in California: findings from a representative, population-based sample of high school students. J Am Acad Child Adolesc Psychiatr 2017; 56(9):739-746.

27. Clark TC, Lucassen MFG, Bullen P, Denny SJ, Fleming TM, Robinson EM, Rossen FV. The health and well -being of transgender high school students: results from the New Zealand Adolescent Health Survey (Youth'12). J Adolesc Health 2014; 55(1):93-99.

28. Zucchi EM, Barros CRS, Redoshi BRL, Deus LFA, Veras MASM. Psychological well-being among transvestites and trans women in the state of São Paulo, Brazil. Cad Saude Publica 2019; 35(3):e00064618.

29. Souza MHT, Malvasi P, Signorelli MC, Pereira PPG. Violence and social distress among transgender persons in Santa Maria, Rio Grande do Sul State, Brazil. Cad Saude Publica 2015; 31(4):767-776.

30. Silva RM, Mangas RMN, Figueiredo AEB, Vieira LJES, Sousa GS, Cavalcanti AMTS, Apolinário AVS. The influence of family problems and conflicts on suicidal ideation and suicide attempts in elderly people. Cien Saude Colet 2015; 20(6):1703-1710.

31. Sheftall AH, Mathias CW, Furr RM, Dougherty DM. Adolescent attachment security, family functioning, and suicide attempts. Attach Hum Dev 2013; 15(4):368-383.

32. Hsu YF, Chen PF, Lung FW. Parental bonding and personality characteristics of first episode intention to suicide or deliberate self-harm without a history of mental disorders. BMC Public Health 2013; 13:421.

33. Bauer GR, Scheim AI, Pyne J, Travers R, Hammond R. Intervenable factors associated with suicide risk in transgender persons: a respondent driven sampling study in Ontario, Canada. BMC Public health 2015; 15(1):1-15.

34. Marshall BD, Socías ME, Kerr T, Zalazar V, Sued O, Arístequi I. Prevalence and Correlates of Lifetime Suicide Attempts Among Transgender Persons in Argentina. J Homosex 2016; 63(7):955-967.

35. Word Health Organization (WHO). Preventing youth violence: an overview of the evidence. Geneva: WHO; 2015.

Artigo apresentado em 04/06/2019 Aprovado em 20/01/2020

Versão final apresentada em22/01/2020

Editores-chefes: Romeu Gomes, Antônio Augusto Moura da Silva 